\title{
Multidimensional quantum dynamics with trajectories: a novel numerical implementation of Bohmian mechanics
}

\author{
Dmitry Nerukh and John H. Frederick \\ Department of Chemistry and Program in Chemical Physics, \\ University of Nevada, Reno, NV 89557, USA \\ Fax: 1775784 6804; email: jhf@chem.unr.edu
}

\begin{abstract}
A novel implementation of the de Broglie-Bohm mechanics is presented. The method employs the use of n-dimensional Delaunay tesselation for the purpose of computing the quantum potential term and is fully generalizable for the multidimensional case. We simulate the scattering of a Gaussian wavepacket from an Eckart barrier in two- and three-dimensions and compare our results against the dynamics obtained using a numerically exact propagation scheme.
\end{abstract}

An alternative interpretation of quantum mechanics, different from the conventional "Copenhagen” view, was introduced by de Broglie in 1927 [1] and later developed into a complete theory by Bohm [2], but has been largely ignored since then. Only recently have workers begun to employ the theory to simulate the dynamics of systems of chemical interest [3-6]. These recent efforts indicate that the de Broglie-Bohm approach is both intuitively attractive in its "causal interpretation” of quantum events [7] and computationally promising. The key reason for this is that quantum phenomena can be described in the framework of hydrodynamic formalism and the time evolution of a system becomes the propagation of "quantum particles” in the Lagrangian representation. 
The postulates of the de Broglie-Bohm theory can be found in original works [2] and in an excellent monograph [7]. The formulas essential for the numerical implementation are outlined here. After representing the wave function in a polar form $\psi=R \mathrm{e}^{i S / \hbar}$, substituting it into the time-dependent Schrödinger equation (TDSE), $i \hbar \frac{\partial \psi}{\partial t}=\left(-\frac{\hbar^{2}}{2 m} \nabla^{2}+V\right) \psi$, and separating real and imaginary parts, one obtains:

$$
\begin{gathered}
\frac{\partial S}{\partial t}+\frac{(\nabla S)^{2}}{2 m}-\frac{\hbar^{2}}{2 m} \frac{\Delta R}{R}+V=0 \\
\frac{\partial R^{2}}{\partial t}+\nabla\left(\frac{R^{2} \nabla S}{m}\right)=0
\end{gathered}
$$

Here $R$ represents the amplitude of the wave function, $S$ represents its phase, and $V$ is a classical potential acting on the system of the mass $m$. Eq. 1 can be thought of as a generalized HamiltonJacobi equation describing the movement of the particle under the influence of a combined potential $Q+V$ [7], where

$$
Q=-\frac{\hbar^{2}}{2 m} \frac{\Delta R}{R}
$$

is an effective "quantum potential".

The particle's equation of motion is then

$$
\frac{d^{2} \mathbf{v}}{d t^{2}}=-\frac{1}{m} \nabla(Q+V)
$$

where $\mathbf{v}=\frac{\nabla S}{m}$ is the velocity of the particle and $\frac{d}{d t}=\frac{\partial}{\partial t}+\mathbf{v} \cdot \nabla$ is the Lagrangian time derivative. Eq. 2 is a continuity equation that defines the equation of motion for the quantum density $\rho=R^{2}$. 
The “quantum potential” $Q$ is a feature that makes the particles' behavior different from the classical one. This time evolving potential is determined by the wave function and expressed through the Laplacian of its amplitude. Computing the latter constitutes the main difficulty in numerical implementations of Bohmian mechanics.

In the Lagrangian formalism the time-dependent wave function can be represented by a set of particletrajectories whose positions define a multidimensional grid. The movement of these particletrajectories causes the grid to be irregular and calculation of the quantum potential $Q$ becomes a challenging task. Most numerical implementations utilize a polynomial interpolation of the grid that can be used for subsequent calculation of the derivatives necessary to calculate $Q$. The use of interpolation makes these methods difficult to extend to higher dimensions. For example, in order to fit a set of points in five dimensions using a third-order polynomial, 54 terms are required. Such a polynomial fit is required at each time step and for higher dimensional problems the size of the polynomial becomes substantially larger. To overcome this difficulty we propose a new numerical method for calculating the derivatives of a function defined on irregular grid. In this method the only limitation on the dimensionality is the intrinsic requirement for any higher-dimensional problem, namely, the number of argument points required to adequately represent the wave function.

When propagating $n$ quantum particles in a $d$-dimensional space we numerically solve the following set of $(2 d+1) n$ ordinary differential equations:

$$
\left\{\begin{aligned}
\dot{x}_{i}^{j} & =v_{i}^{j} & & \\
\dot{v}_{i}^{j} & =\frac{1}{m} F_{i}^{j} & & i=1 \ldots d \\
\dot{\rho}^{j} & =-\nabla \cdot \mathbf{v}^{j} \rho^{j} & &
\end{aligned}\right.
$$


where $\mathbf{x}^{j}$ are the particles' coordinates and

$$
\mathbf{F}^{j}=-\nabla(Q+V)
$$

are forces acting on the particles.

Note that the phase of the wave function $S$ enters into the equations of motion only through its gradient, that is, the velocity of the particles. To obtain the velocity, one must compute the gradient of the potential in order to determine the forces acting on the particle. Hence, we need to calculate numerical derivatives three times: in formulas 3, 5c, and 6 . This situation turns out to be very unfavorable computationally, generally leading to an exponentially rapid accumulation of errors. It is possible, however, to reduce the number of numerical derivatives to two. Assuming a weak timedependence of $\nabla \mathbf{v}$, Eq. 5c can be explicitly integrated providing an analytical equation of motion for the quantum density:

$$
\rho(t+d t)=\rho(t) e^{-\nabla \mathbf{v} d t}
$$

Thus, using (4) and (7), a recurrence relation for the velocity gradient $[-\nabla \mathbf{v}]$ gives:

$$
\rho(t+d t)=\rho(t) \exp \left[-\nabla \mathbf{v}(t-d t)+\frac{1}{m} \Delta V(t) d t+\frac{1}{m} \Delta Q(t) d t\right] d t
$$

One may address the problem of evaluating $\mathbf{F}$ by interpolating between the particles trajectories to construct a rectangular grid representation of $\psi$. Our algorithm differs from this standard approach by utilizing the irregular mesh of trajectory points directly. "Delaunay triangulation” ("Delaunay tesselation" for higher dimensionality) [8] is then used to organize this irregular grid for the purpose of computing $\mathbf{F}$. Once the triangulation is built, the points of intersection of the line parallel to the derivative axis with the edges of the triangles constituting the mesh can be found (Fig. 1). With the help of such a procedure we thus construct points lying approximately on the surface of the function. 
The points can then be fitted by a one-dimensional third-order polynomial that can be used for calculating derivatives of the function.

A simplex in d-dimensional space is a structure formed by joining by segments all possible combinations of $d+1$ points. This is the "simplest" combination of points in d-dimensional space enclosing a finite volume. In 2D this is a triangle, in 3D a tetrahedron. Planar triangulation is a division of a set of points into adjacent triangles with vertices from this set. Triangulation can be done in many ways, with some ways being more useful for numerical analysis that others. The Delaunay triangulation has the following important property: inside the circumference of any triangle there are no other points of this set. Therefore such a triangulation is "natural" and optimal in many respects. It is relatively easy to construct and there are many known algorithms which can be used to efficiently construct such a triangulation (mostly developed for low-dimensional cases, however). This concept can easily be generalized to a d-dimensional set.

The algorithm can be described by the following steps.

1. Construct Delaunay tesselation using the particle trajectory points;

2. For each point compile a list of simplices to which the point belongs;

3. For each degree of freedom find the intersections of the line passing through the point and parallel to the coordinate axes (derivative line) with the edges of two simplices lying on the opposite sites of the point (Fig. 1);

4. Map these intersection points up to the approximated function surface (Fig. 1);

5. Repeat this procedure in both directions until a desired number of intersection points (normally up to 10) is found; 
6. The points of intersection form an approximated function cut in the direction of derivative - fit this one-dimensional cut by a third-order polynomial;

7. Calculate the necessary derivatives from the polynomial.

There are two essential, but time-consuming steps in this scheme. The first is the procedure of finding which simplex edges are intersected by the derivative line (step 3). This is, in fact, the general problem of a segment - polygon intersection. The solution for the 3D intersection of a segment and a triangle is based on the concept of the signed volume of a tetrahedron and can be straightforwardly generalized for d-dimensional space [9]. The whole algorithm for 3D reduces to the calculation of five fourthorder determinants. Note that both this step and the tesselation can be very effectively accomplished using exact arithmetic. Specifically, we used the algorithm by Clarkson [11] for the Delaunay tesselation.

The second important step is the calculation of the cut of the function along the derivative line that passes through the point at which the derivative value is needed. In fact, we have to calculate the coordinates of the point of intersection of a plane passing through the point $\left(x_{0}, y_{0}, z_{0}, \ldots\right)$ and parallel to the derivative axes and the edge of the simplex (Fig. 2). For the 3D case, this will be the intersection of the plane defined by the equation $y=y_{0}$ and the line passing through the points $\left(x_{1}, y_{1}, z_{1}\right)$ and $\left(x_{2}, y_{2}, z_{2}\right)$ (Fig. 2a). The equation describing the latter line can be represented by a vector $\mathbf{a}=\mathbf{r}_{2}-\mathbf{r}_{1}$ that passes through the point $\mathbf{r}_{1}: \mathbf{r}-\mathbf{r}_{1}=\mathbf{a}$, where the vectors are defined by $\mathbf{r}_{i}=\left(x_{i}, y_{i}, z_{i}\right)$. Solving the set of linear equations

$$
\left\{\begin{array}{c}
y=y_{0} \\
x-x_{1}=\left(x_{2}-x_{1}\right) t \\
y-y_{1}=\left(y_{2}-y_{1}\right) t \\
z-z_{1}=\left(z_{2}-z_{1}\right) t
\end{array}\right.
$$


the coordinates of the desired point $(x, y, z)$ are obtained.

For a 4D problem this point is defined by intersection of the hyperplane $\left\{\begin{array}{l}y=y_{0} \\ z=z_{0}\end{array}\right.$ and a plane passing through three points $\left(x_{1}, y_{1}, z_{1}\right),\left(x_{2}, y_{2}, z_{2}\right)$, and $\left(x_{3}, y_{3}, z_{3}\right)$ (Fig. 2b). This plane is defined by parametric equations connecting two arbitrary vectors lying in the plane $\mathbf{a}=\mathbf{r}_{2}-\mathbf{r}_{1}$ and $\mathbf{b}=\mathbf{r}_{3}-\mathbf{r}_{1}$, and the point $\left(x_{1}, y_{1}, z_{1}\right): \mathbf{r}-\mathbf{r}_{1}=\mathbf{a} w+\mathbf{b} v$. The set of equations defining the point of intersection is

$$
\left\{\begin{array}{l}
y=y_{0} \\
z=z_{0} \\
x-x_{1}=\left(x_{2}-x_{1}\right) w+\left(x_{3}-x_{1}\right) v \\
y-y_{1}=\left(y_{2}-y_{1}\right) w+\left(y_{3}-y_{1}\right) v \\
z-z_{1}=\left(z_{2}-z_{1}\right) w+\left(z_{3}-z_{1}\right) v \\
u-u_{1}=\left(u_{2}-u_{1}\right) w+\left(u_{3}-u_{1}\right) v
\end{array}\right.
$$

For the general $d$-dimensional case, after substituting $y_{0}, z_{0}, \ldots$ into the last three $(d-1)$ equations, the set becomes

$$
\left\{\begin{array}{lllllll}
a_{1}\left(r_{2}^{1}-r_{1}^{1}\right) & +a_{2}\left(r_{3}^{1}-r_{1}^{1}\right) & +\ldots & +a_{d-2}\left(r_{d-1}^{1}-r_{1}^{1}\right) & -r^{1} & +0 & =-r_{1}^{1} \\
a_{1}\left(r_{2}^{2}-r_{1}^{2}\right) & +a_{2}\left(r_{3}^{2}-r_{1}^{2}\right) & +\ldots & +a_{d-2}\left(r_{d-1}^{2}-r_{1}^{2}\right) & +0 & +0 & =r_{0}^{2}-r_{1}^{2} \\
\vdots & \vdots & \vdots & \vdots & \vdots & \vdots & \vdots \\
a_{1}\left(r_{2}^{i}-r_{1}^{i}\right) & +a_{2}\left(r_{3}^{i}-r_{1}^{i}\right) & +\ldots & +a_{d-2}\left(r_{d-1}^{i}-r_{1}^{i}\right) & +0 & +0 & =r_{0}^{i}-r_{1}^{i} \\
\vdots & \vdots & \vdots & \vdots & \vdots & \vdots & \vdots \\
a_{1}\left(r_{2}^{d}-r_{1}^{d}\right) & +a_{2}\left(r_{3}^{d}-r_{1}^{d}\right) & +\ldots & +a_{d-2}\left(r_{d-1}^{d}-r_{1}^{d}\right) & +0 & -r^{d} & =-r_{1}^{d}
\end{array},\right.
$$

where $r_{i}^{j}$ is the $\mathrm{j}$ coordinate of the vector $\mathbf{r}_{i}$ and $a_{i}$ - the parameter. 
Solution of this set of linear equations gives $d-2$ coefficients $a$ and two coordinates of the point of intersection: $r^{1}$ and $r^{d}$ (the other $d-2$ coordinates are equal to the corresponding coordinates of the $\mathbf{r}_{0}$ point).

Another serious problem arises in the treatment of boundary points, especially those that are at the “corners” of the argument space. In the worst cases the derivative lines do not intersect any simplex. To handle these situations the derivative line is set not exactly parallel to the derivative axis but slightly turned in such a way as to intersect some boundary simplices. The angle of turning then can be varied and statistics accumulated in order to improve the accuracy. Despite these technical challenges, the present approach makes the calculation of derivatives of high dimensional functions feasible since it does not involve prohibitively large polynomial fitting for the construction of a regular mesh.

The scattering of a Gaussian wavepacket from an Eckart barrier in two- and three-dimensional space is chosen as a representative example of an event with strong quantum character that cannot be modeled by purely classical methods. The model potential surface is an Eckart potential along the $\mathrm{x}$ axis with a harmonic oscillator in the y- and z-directions:

$$
V(x, y)=V_{0} \operatorname{sech}^{2}\left[a\left(x-x_{b}\right)\right]+b\left(y-y_{b}\right)^{2}+b\left(z-z_{b}\right)^{2}
$$

In the two-dimensional model, we omit the z-coordinate dependence.

Fig. 3 presents snapshots of the propagation of two-dimensional system using 386 particles of unit mass with initial kinetic energy 20 a.u. The time propagation is carried out for a total time of 1.1 a.u. using time steps of 0.01 a.u. The barrier is defined by $V_{0}=20, a=1$ and $x_{b}=6$. The wavepacket is initially placed at the point $(2,2)$ and has initial momentum directed towards the barrier along the $\mathrm{x}$ axis. The resulting behavior of the system exhibits the expected quantum effects: the wavepacket is split by the barrier and part of the quantum density transmits through the barrier even though the initial 
kinetic energy of the particles and the barrier height are equal. The reliability of the simulation is tested by comparing to a numerically exact propagation using FFT/grid method [10] which is shown on the left of Fig. 3. Despite minor differences at longer times the overall behavior of the wavepacket, including the ratio of the heights of reflected and transmitted peaks is represented well by the present method.

It is worth noting that the number of particles used in the present approach (386) is much lower than that used in the FFT/grid method (128 by 128 grid makes 16384 nodes). This partly accounts for the difference in quality of the simulations. The effect is particularly noticeable after the splitting of the wavepacket. After spreading the particles and pushing the quantum density to the wings of the wavepacket, one is left with only a few particles in the vicinity of the peak maxima (Fig. 3 IIc). These few particles are not sufficient for an accurate calculation of the derivatives and the behavior of the system quickly accumulates errors. Nevertheless, even with such a small number of points the agreement can be considered very good, at least at times before and shortly after splitting.

Another visible discrepancy between the present and the exact methods is in the shape of the transmitted part. In the present method this part is less elongated, though of approximately the same height as the numerically exact packet. This effect is likely due to the assumption of constant $\nabla \mathbf{v}$ and the use of Eq. (7) in place of the exact Eq. (2). The resulting approximate dynamics introduces systematic errors that are not eliminated by increasing the number of particles used. Using the exact set of equations (5) fixes this problem, but at the expense of allowing a much faster accumulation of errors.

This last discrepancy also explains the differences in the transmission probability for both methods, shown in Fig. 4. Here, the probability is computed as a ratio of the integrals calculated under two areas on left and right sides of the line drawn through the barrier center. Although the final transmitted 
probability is well-approximated by the present method, this probability accumulates more slowly and peaks later than exact result.

The present method has also been applied to a three-dimensional Eckart barrier transmission. The shape of the barrier is the same as in the two-dimensional case, the only difference being an additional harmonic term along the $\mathrm{z}$ axis. The number of particles used, 6270, is approximately equal to the number of particles per degree of freedom for the two-dimensional case and the results are presented in Fig. 5. In order to display the quantum density the diameters of the three-dimensional points were chosen to be proportional to the value of the quantum density carried by the particles. In fact, only the points located in the proximity of the peak maximum are visible. This way of plotting reveals the behavior of the quantum particles with time. The distribution is spherical at the start, then flattens in front of the barrier plane, soon followed by some of the particles breaking through the barrier and forming the transmitted distribution, of smaller height. The reflected part reverses direction and even exhibits self-interference in exact accordance with quantum theory (the self-interference of the reflected part was also observed in 2D simulation).

Summarizing, a novel method for simulating the dynamics of a fully quantum system in the framework of de Broglie-Bohm mechanics is presented and has been used to simulate barrier transmission dynamics in comparison with exact results. The method has several intrinsic advantages, primarily: (i) it is totally generalizable arbitrary dimensions; and (ii) computational expenses are expected to grow slower than exponential with number of degrees of freedom. There are also several ways one might improve the effectiveness of the algorithm, for example, by using alternative algorithms from welldeveloped areas of computational geometry (for building Delaunay tesselation), classical molecular dynamics, and hydrodynamics (for propagating trajectories). Also the approach naturally incorporates the possibility of constructing various mixed quantum-classical methods. We are presently engaged in pursuing these avenues of investigation. 
The authors thank Prof. Robert E. Wyatt for helpful discussions and samples of his code for propagating one-dimensional quantum trajectories. This work has been supported by the National Science Foundation under grant CHE-9727562.

\section{References}

1. Electrons et Photons. Rapports et Discussions du Cinquieme Conseil de Physique, 1927, GauthierVillars, Paris, 1928.

2. D. Bohm, Phys. Rev. 85 (1952) 166; D. Bohm, Phys. Rev. 85 (1952) 180.

3. C.L. Lopreore and R.E. Wyatt, Phys. Rev. Lett. 82 (1999) 5190;

4. R.E. Wyatt, J. Chem. Phys. 111 (1999) 4406;

5. R.E. Wyatt, D.J. Kouri, and D.K. Hoffman, J. Chem. Phys. 112 (2000) 10730.

6. F.S. Mayor, A. Askar, and H.A. Rabitz, J. Chem. Phys. 111 (1999) 2423.

7. P.R. Holland, The quantum theory of motion, Cambridge University Press, 1993.

8. F.P. Preparata and M.I. Shamos, Computational geometry. An introduction, Springer-Verlag, 1985.

9. J. O'Rourke, Computational Geometry in C, Cambridge Univ. Press, 1994.

10. R. Kosloff, Annu. Rev. Phys. Chem. 45 (1994) 145.

11. http://cm.bell-labs.com/who/clarkson. 


\section{Figure captions}

1. Delaunay tesselation of the argument points and mapping the line along the derivative axis onto the tesselation structure. The points of intersection of the upper line and edges of the triangles are used to approximate the cut of the surface.

2. Calculation of the coordinates of the point of intersection of the derivative line with the edge of a simplex in 2D and 3D case. The point $\left(x_{0}, y_{0}, f\left(x_{0}, y_{0}\right)\right)\left[\left(x_{0}, y_{0}, z_{0}, f\left(x_{0}, y_{0}, z_{0}\right)\right)\right]$ is a point at which the derivative is calculated, $(x, y, f(x, y))[(x, y, z, f(x, y, z))]$ is the intersection point, $\left(x_{i}, y_{i}, f\left(x_{i}, y_{i}\right)\right)\left[\left(x_{i}, y_{i}, z_{i}, f\left(x_{i}, y_{i}, z_{i}\right)\right)\right] \mathrm{i}=1 . .3$ are the vertices of the intersected simplex's face.

3. Propagation of the quantum density $\rho$ of 2D Gaussian wavepacket on the Eckart barrier-harmonic potential surface by a numerically exact method (left column - I) and by the present method (right column - II). Snapshots at 0 (a), 0.8 (b), and 0.95 (c) a.u. are shown. The system consists of 386 particles of 1 a.u. mass. The initial velocity of the particles was directed along the $\mathrm{x}$ axes and their initial kinetic energy was equal to the height of the barrier (20 a.u.). The continuous surfaces and their contour lines drawn on the right plots are for visualization purpose only. They are an artificial interpolation of the real particles shown above the surfaces.

4. Time-dependence of the probability of the transmission of quantum density through the Eckart barrier for 2D case. Circles correspond to the exact propagation, squares represent the present method.

5. Propagation of 3D Gaussian wavepacket on the Eckart barrier-harmonic potential surface. Snapshots at 0 (a), 108 (b), and 173 (c) a.u. are shown. The radii of the points are proportional to the quantum density carried by the particles. The system consists of 6270 particles of 2000 a.u. mass. The initial velocity of the particles was directed along the $\mathrm{x}$ axes and their initial kinetic energy, 3.31 a.u., was slightly below the height of the barrier (3.65 a.u.). 


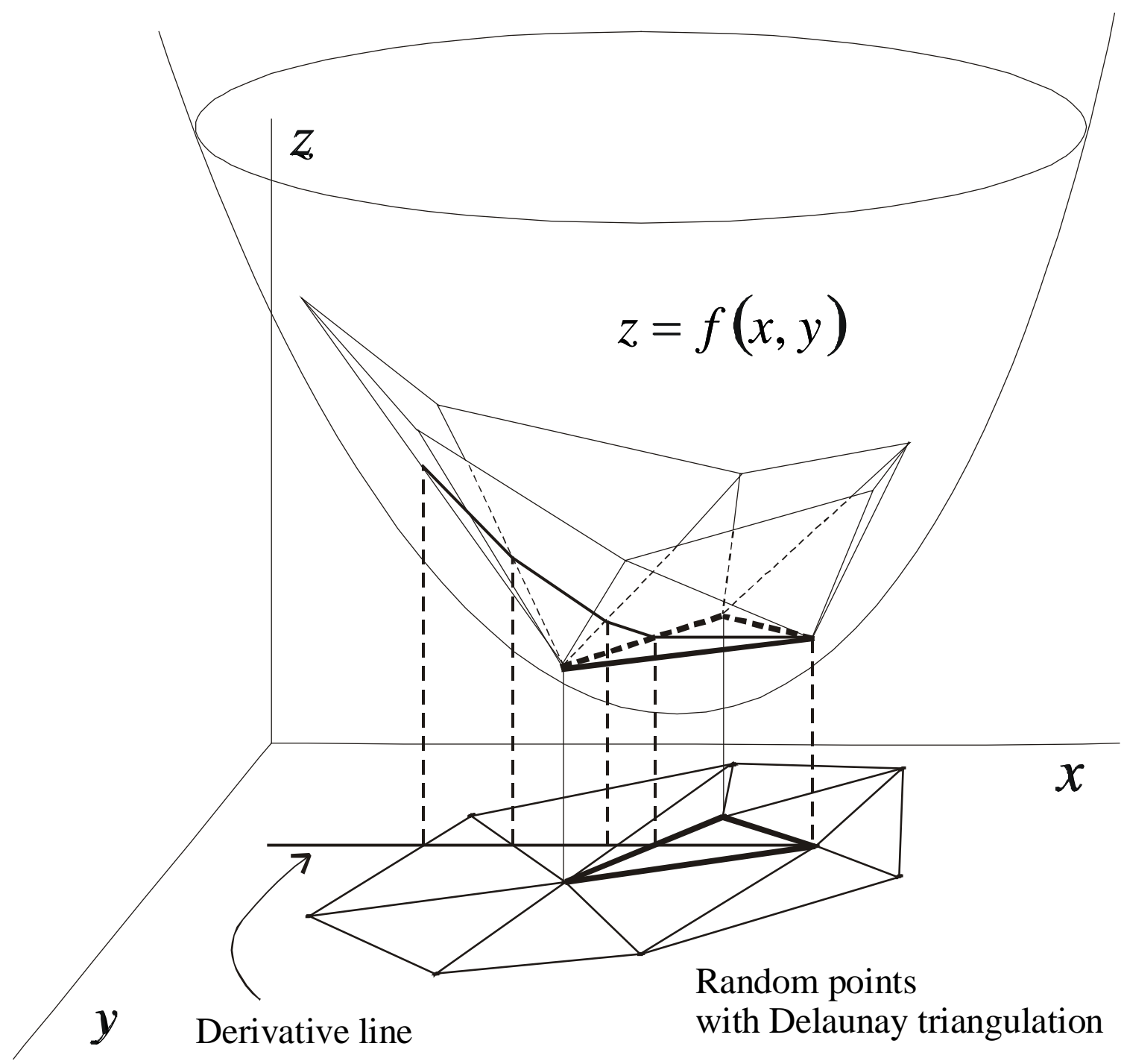

Fig. 1. Delaunay tesselation of the argument points and mapping the line along the derivative axis onto the tesselation structure. The points of intersection of the upper line and edges of the triangles are used to approximate the cut of the surface. 


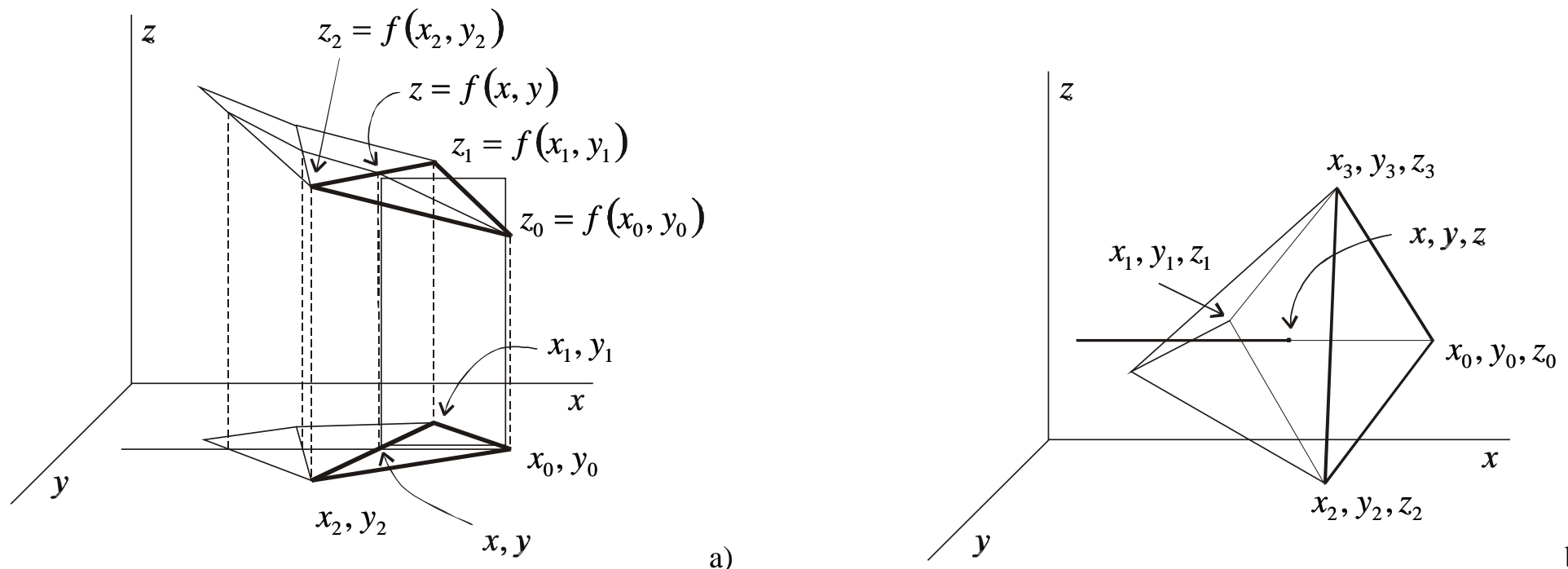

Fig. 2. Calculation of the coordinates of the point of intersection of the derivative line with the edge of a simplex in 2D and 3D case. The point $\left(x_{0}, y_{0}, f\left(x_{0}, y_{0}\right)\right)\left[\left(x_{0}, y_{0}, z_{0}, f\left(x_{0}, y_{0}, z_{0}\right)\right)\right]$ is a point at which the derivative is calculated, $(x, y, f(x, y))[(x, y, z, f(x, y, z))]$ is the intersection point, $\left(x_{i}, y_{i}, f\left(x_{i}, y_{i}\right)\right)\left[\left(x_{i}, y_{i}, z_{i}, f\left(x_{i}, y_{i}, z_{i}\right)\right)\right] \mathrm{i}=1 . .3$ are the vertices of the intersected simplex's face. 


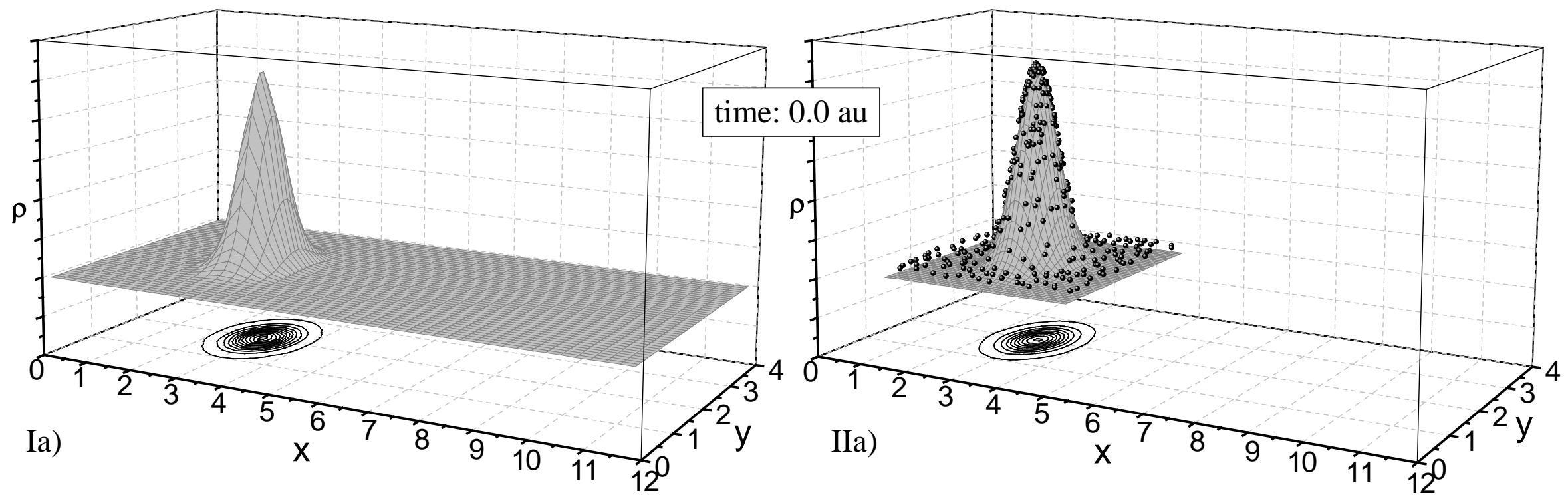

Fig. 3a. Propagation of the quantum density $\rho$ of 2D Gaussian wavepacket on the Eckart barrier-harmonic potential surface by a numerically exact method (left column - I) and by the present method (right column - II). Snapshots at 0 (a), 0.8 (b), and 0.95 (c) a.u. are shown. The system consists of 386 particles of 1 a.u. mass. The initial velocity of the particles was directed along the $\mathrm{x}$ axes and their initial kinetic energy was equal to the height of the barrier (20 a.u.). The continuous surfaces and their contour lines drawn on the right plots are for visualization purpose only. They are an artificial interpolation of the real particles shown above the surfaces. 


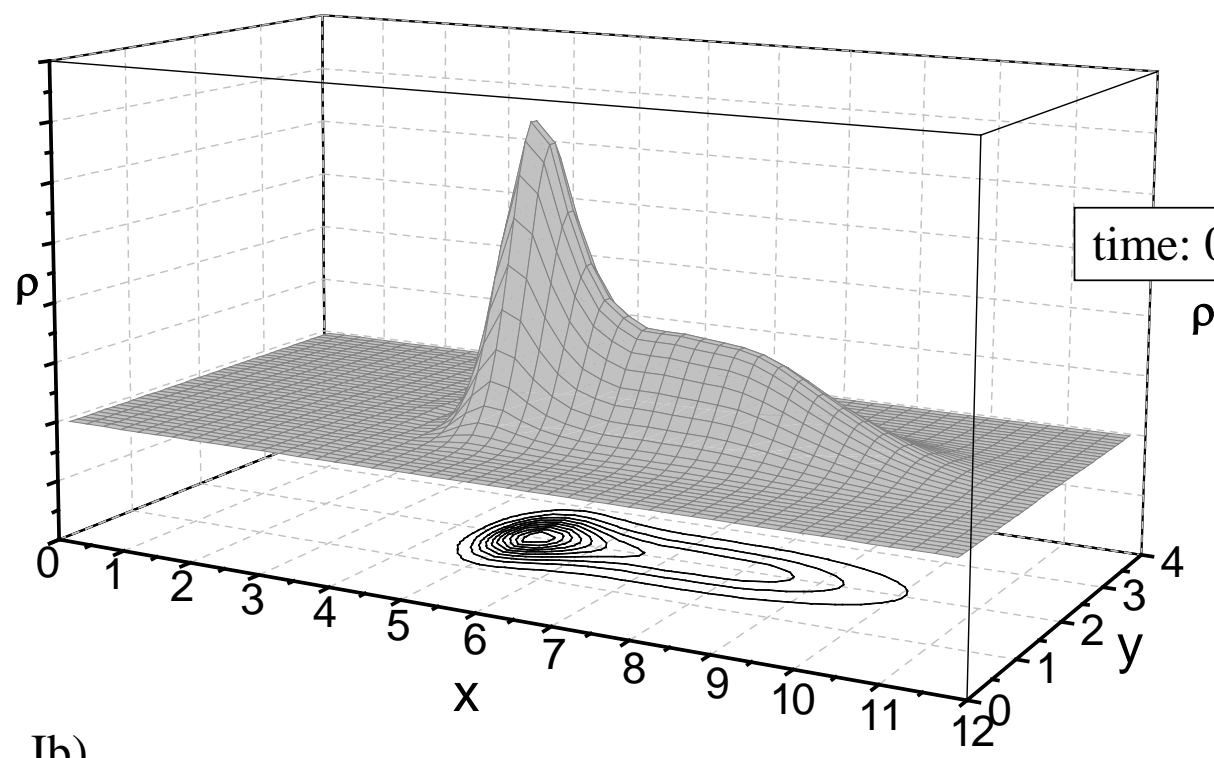

$\mathrm{Ib})$

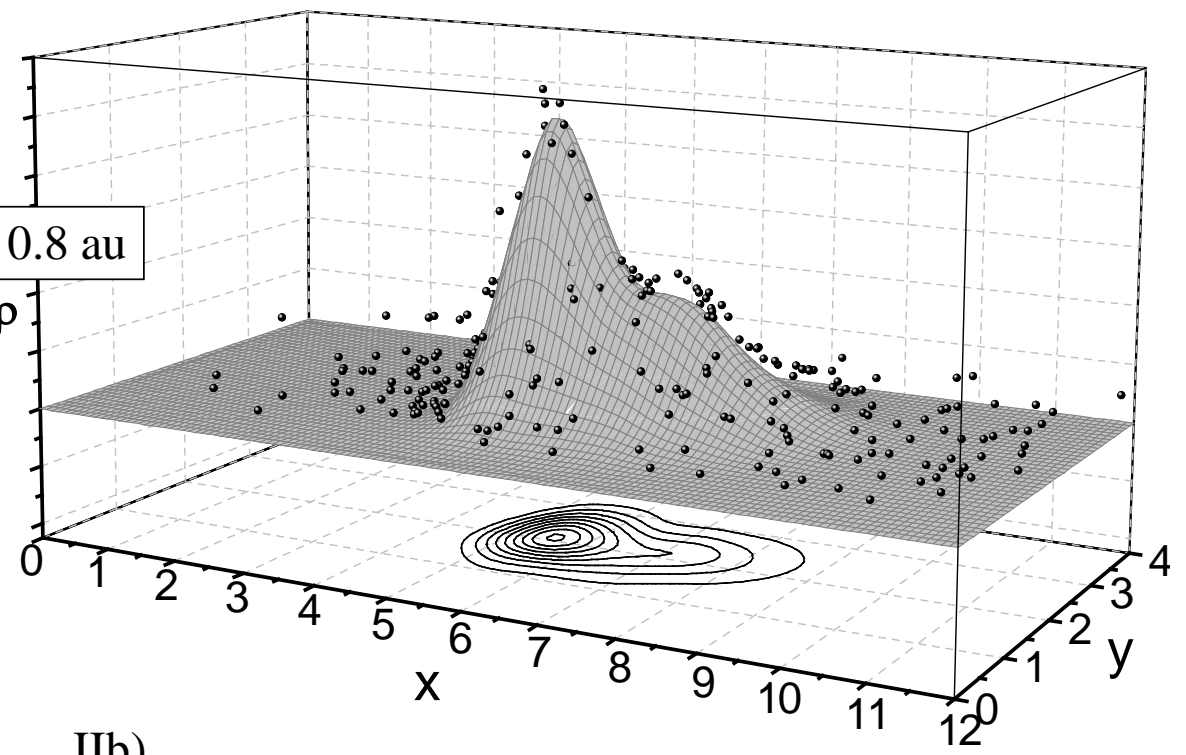

IIb)

Fig. 3b. Propagation of the quantum density $\rho$ of 2D Gaussian wavepacket on the Eckart barrier-harmonic potential surface by a numerically exact method (left column - I) and by the present method (right column - II). Snapshots at 0 (a), 0.8 (b), and 0.95 (c) a.u. are shown. The system consists of 386 particles of 1 a.u. mass. The initial velocity of the particles was directed along the $x$ axes and their initial kinetic energy was equal to the height of the barrier (20 a.u.). The continuous surfaces and their contour lines drawn on the right plots are for visualization purpose only. They are an artificial interpolation of the real particles shown above the surfaces. 


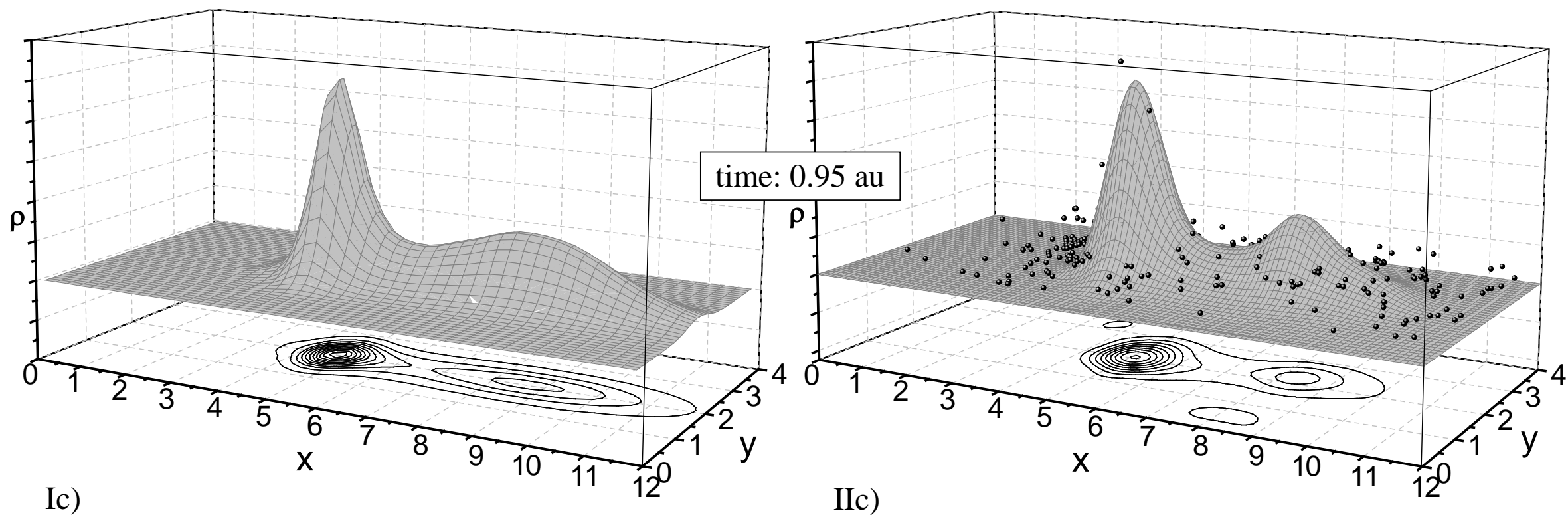

Fig. 3c. Propagation of the quantum density $\rho$ of 2D Gaussian wavepacket on the Eckart barrier-harmonic potential surface by a numerically exact method (left column - I) and by the present method (right column - II). Snapshots at 0 (a), 0.8 (b), and 0.95 (c) a.u. are shown. The system consists of 386 particles of 1 a.u. mass. The initial velocity of the particles was directed along the $x$ axes and their initial kinetic energy was equal to the height of the barrier (20 a.u.). The continuous surfaces and their contour lines drawn on the right plots are for visualization purpose only. They are an artificial interpolation of the real particles shown above the surfaces. 


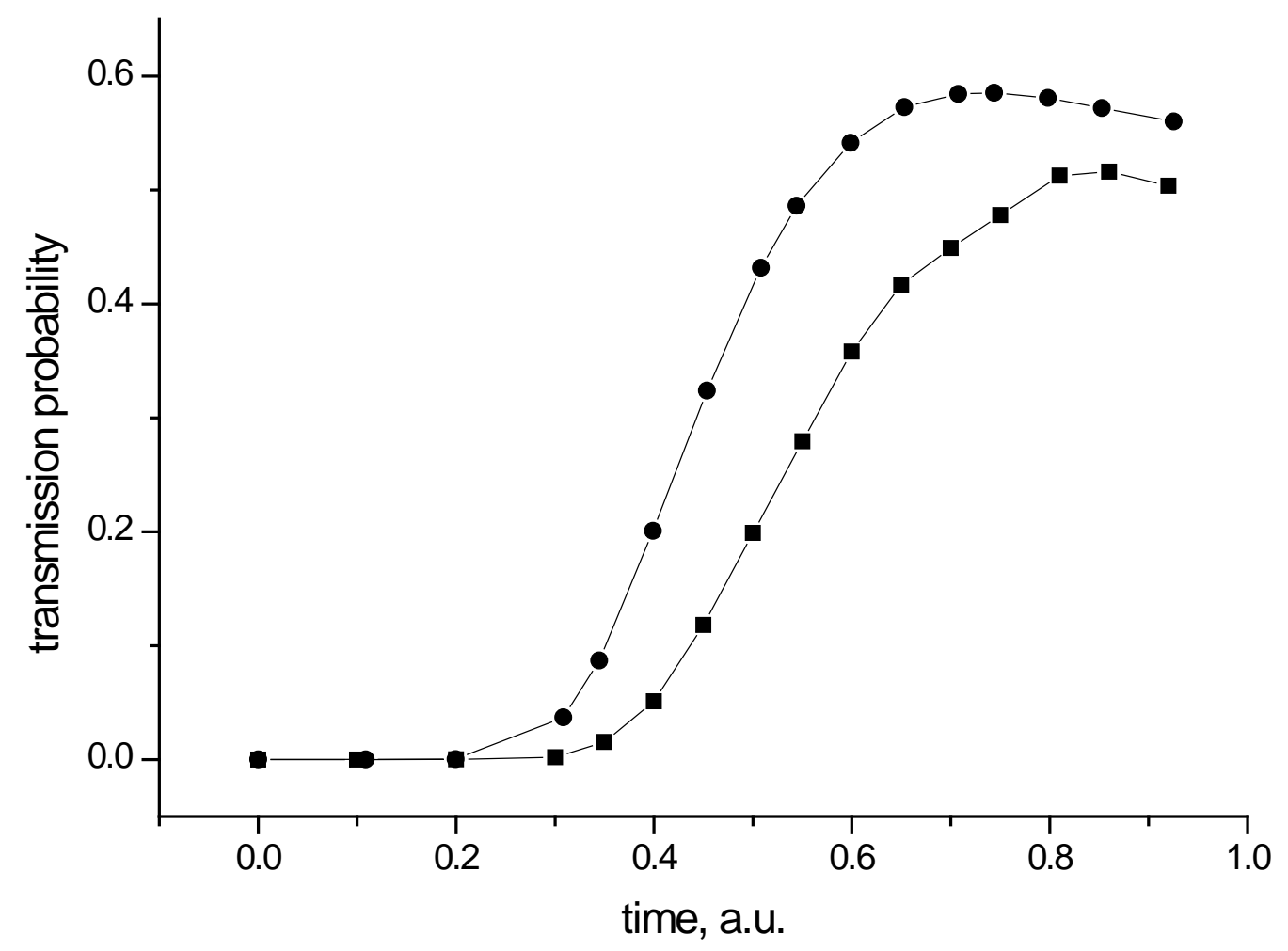

Fig. 4. Time-dependence of the probability of the transmission of quantum density through the Eckart barrier for 2D case. Circles correspond to the exact propagation, squares represent the present method. 



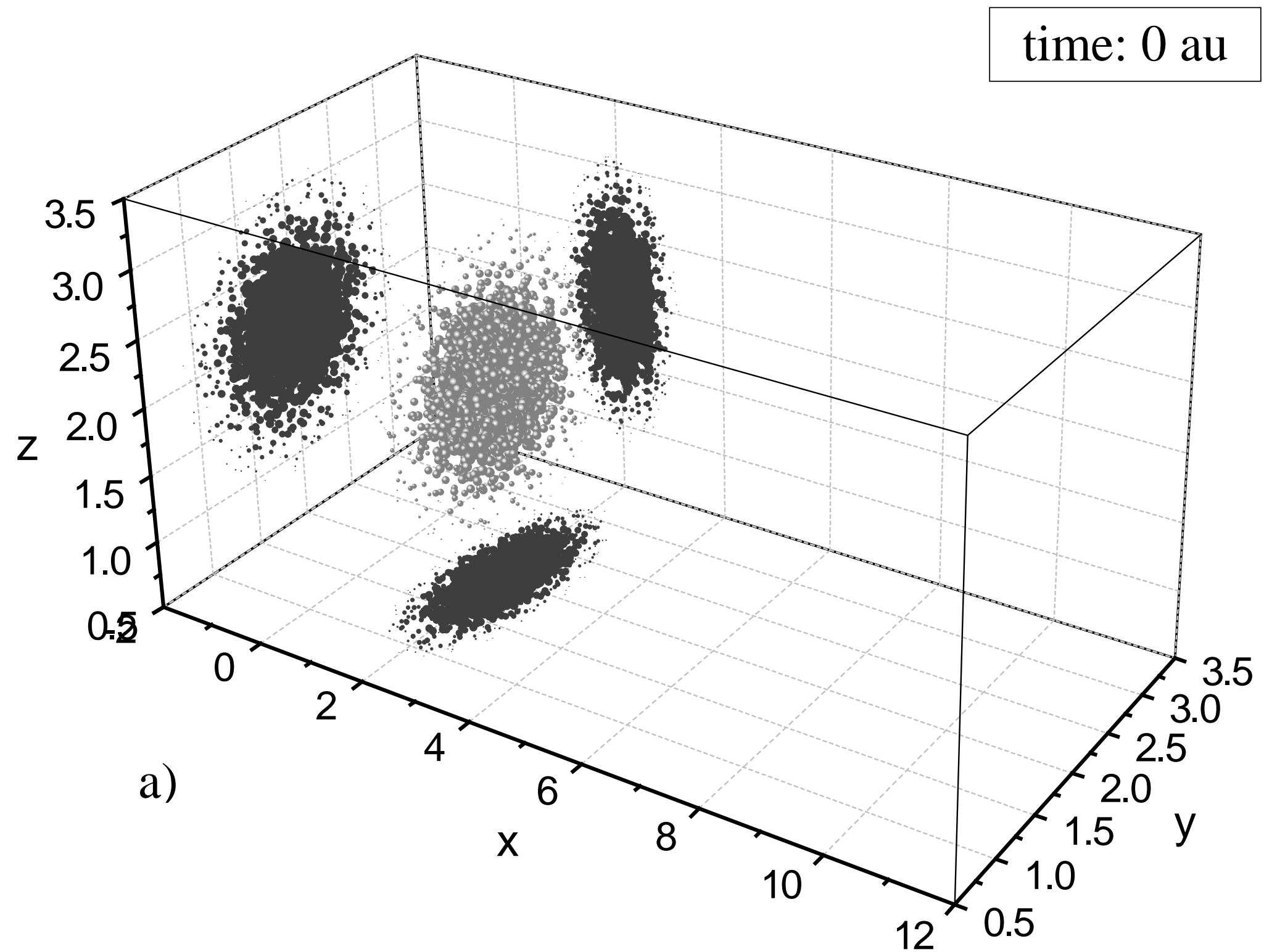

Fig. 5a. Propagation of 3D Gaussian wavepacket on the Eckart barrier-harmonic potential surface. Snapshots at 0 (a), 108 (b), and 173 (c) a.u. are shown. The radii of the points are proportional to the quantum density carried by the particles. The system consists of 6270 particles of 2000 a.u. mass. The initial velocity of the particles was directed along the $\mathrm{x}$ axes and their initial kinetic energy, 3.31 a.u., was slightly below the height of the barrier (3.65 a.u.). 


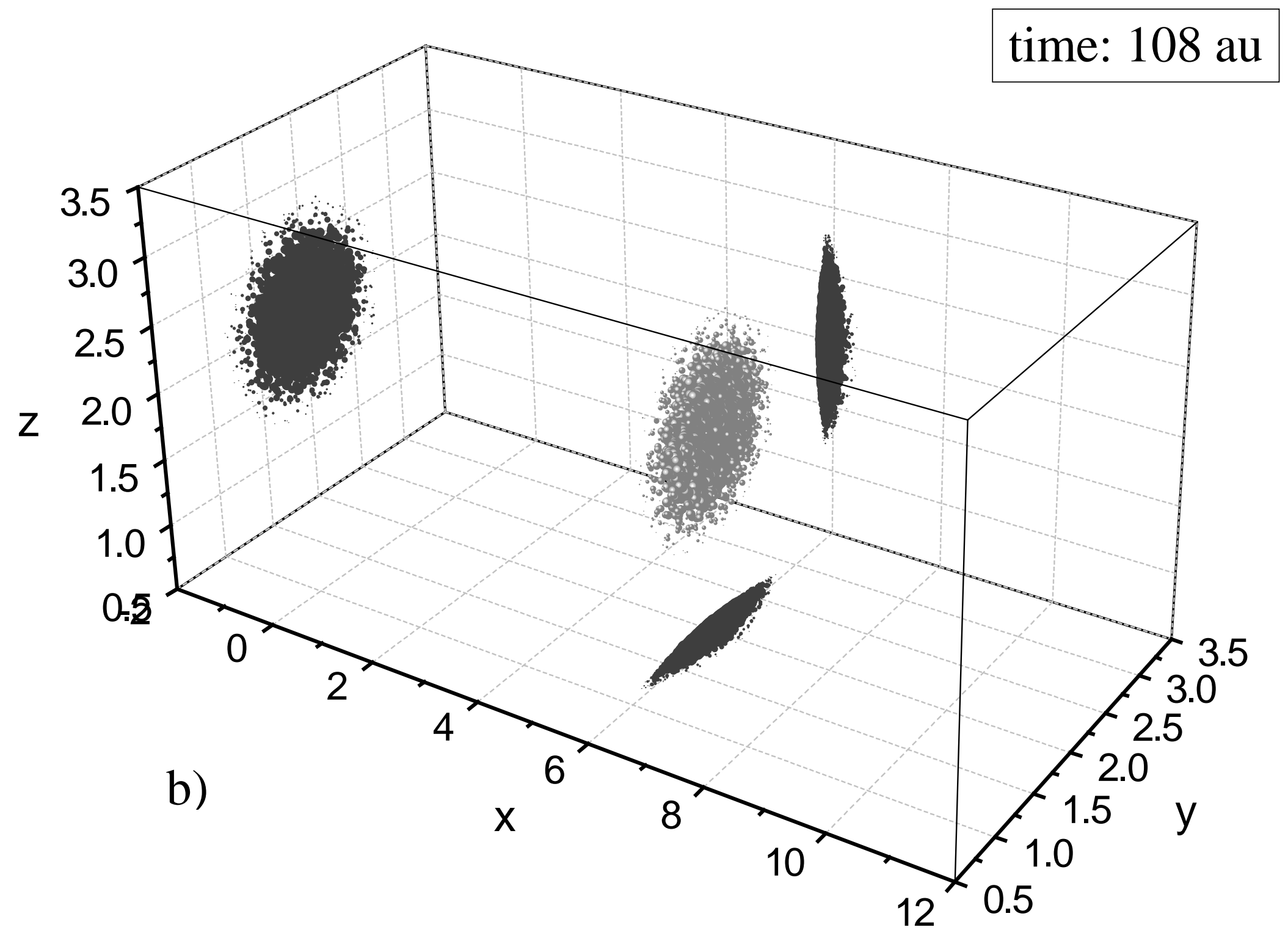

Fig. 5b. Propagation of 3D Gaussian wavepacket on the Eckart barrier-harmonic potential surface. Snapshots at 0 (a), 108 (b), and 173 (c) a.u. are shown. The radii of the points are proportional to the quantum density carried by the particles. The system consists of 6270 particles of 2000 a.u. mass. The initial velocity of the particles was directed along the $\mathrm{x}$ axes and their initial kinetic energy, 3.31 a.u., was slightly below the height of the barrier (3.65 a.u.). 


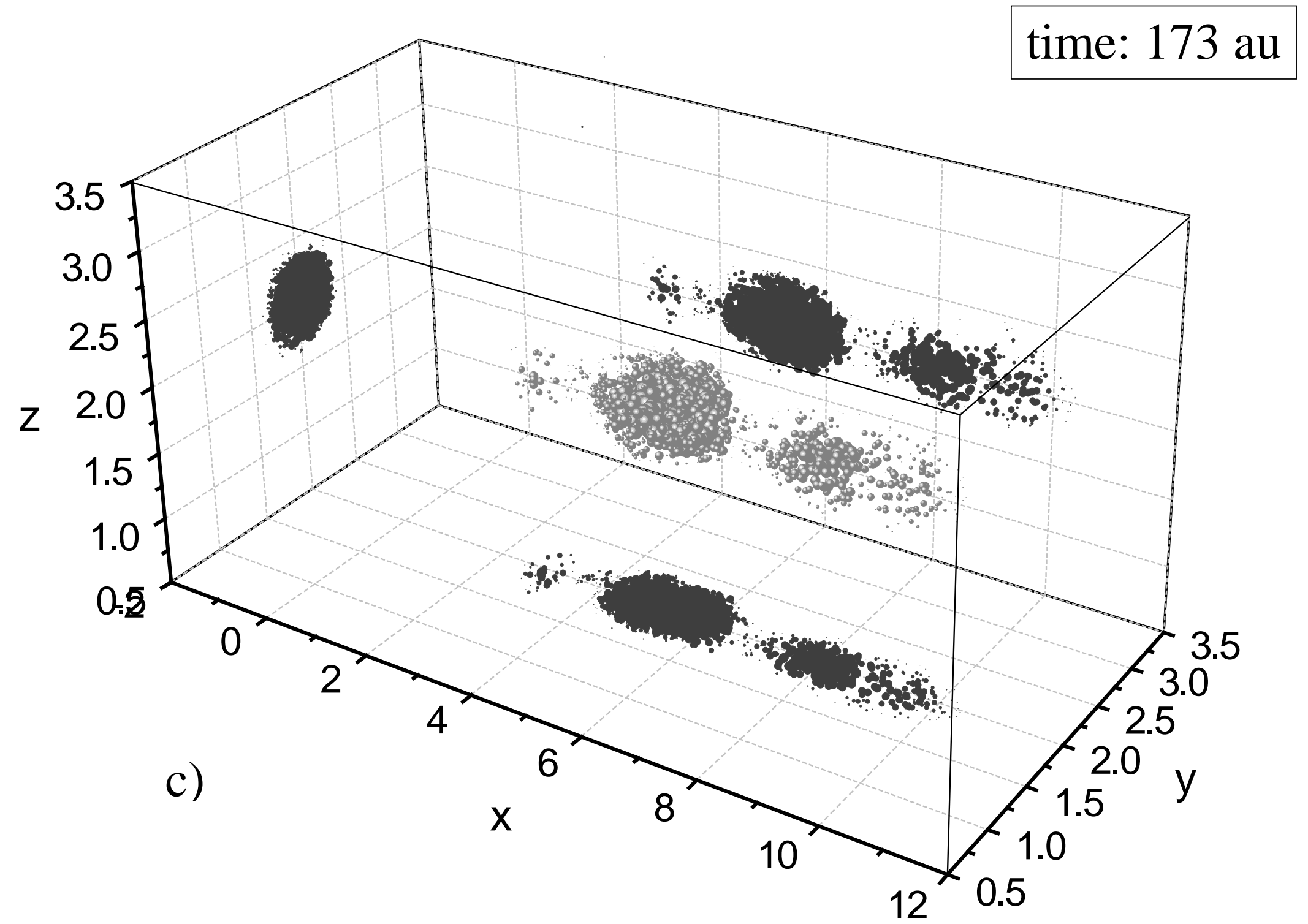

Fig. 5c. Propagation of 3D Gaussian wavepacket on the Eckart barrier-harmonic potential surface. Snapshots at 0 (a), 108 (b), and 173 (c) a.u. are shown. The radii of the points are proportional to the quantum density carried by the particles. The system consists of 6270 particles of 2000 a.u. mass. The initial velocity of the particles was directed along the $x$ axes and their initial kinetic energy, 3.31 a.u., was slightly below the height of the barrier (3.65 a.u.). 